\title{
Rheological Properties of Mixtures of Oppositely Charged Polyelectrolytes. A Study of the Interactions between a Cationic Cellulose Ether and a Hydrophobically Modified Poly[sodium 2-(acrylamido)-2-methylpropanesulfonate]
}

\author{
Roger C. W. LIU, ${ }^{* * *}$ Yotaro MoRISHIMA, ${ }^{* *}$ and Françoise M. WINNIK*,† \\ ${ }^{*}$ Faculty of Pharmacy and Department of Chemistry, University of Montréal, \\ C. P. 6128 succursale Centre-ville, Montréal QC H3C 3J7, Canada \\ ${ }^{* *}$ Department of Macromolecular Science, Graduate School of Science, Osaka University, \\ Toyonaka, Osaka 560-0043, Japan
}

(Received November 8, 2001; Accepted February 7, 2002)

\begin{abstract}
Mixed solutions of a cationic cellulose ether (JR400) and, either poly[sodium 2-(acrylamido)-2methylpropanesulfonate] (PAMPS), or a copolymer of sodium 2-(acrylamido)-2-methylpropanesulfonate and $N-n$ dodecylmethacrylamide (PAMPS-Dod) in the semi-dilute regime $\left(10 \mathrm{~g} \mathrm{~L}^{-1}\right)$ have been examined by oscillatory shear and steady shear measurements. Mixtures consisting of the polycation as major component and PAMPS-Dod as minor component, were viscous fluids at low polyanion concentration and became gel-like fluids with increasing polyanion content. The zero shear viscosity was a strong function of polyanion content, increasing significantly with polyanion content. The fluids are viscoelastic, exhibit Newtonian behavior at sufficiently low shear rates and become shear thinning at higher shear rates. Steady shear viscosities were fitted with the Carreau-Yasuda model yielding the time constant associated with the relaxation process. The rheological parameters concurred in support of an association model involving the formation of a crosslinked network between two oppositely charged polyelectrolytes differing greatly in molecular architecture, charge density, and conformation.
\end{abstract}

KEY WORDS Interpolyelectrolyte Complex / Rheology / Sodium 2-Acrylamido-2-methylpropanesulfonate / Transient Network / Polysaccharide / Viscoelasticity /

A great variety of consumer products, such as cosmetics, drug formulations, foodstuff or paints rely on the ability of water-soluble polymers to control the rheology of fluids. ${ }^{1-3}$ Generally, the polymer is not used alone, but in combination with other polymers or surfactants and it is the synergic action of the components that create the required rheological properties. Of particular interest are systems containing oppositelycharged polymers and surfactants, which form complexes as a result of electrostatic interactions between the charged head group of the surfactant and the ionized units of the polymer, and, in some cases, additional hydrophobic interactions. ${ }^{4}$ Mixtures of cationic cellulose ether derivatives and oppositely charged surfactants have been investigated extensively, ever since Goddard reported that the addition of a surfactant, such as sodium dodecylsulfate (SDS), to aqueous cationic cellulose ethers leads to a very large increase in solution viscosity or, in some cases, to phase separation. ${ }^{5}$ Further studies have indicated that fluids containing cationic cellulose ethers and anionic surfactants are viscoelastic in given situations and exhibit shear thinning properties that depend in a subtle way on the molecular weight of the polymer and on the nature of the surfactant. ${ }^{6,7}$ The surfactant-triggered changes in viscosity of polyelectrolyte solutions have been attributed either to electrostatic interactions between the charged groups or to hydrophobic interactions between the nonpolar tails of the surfactant and hydrophobic patches along the polysaccharide backbone, or to an interplay of both mechanisms. ${ }^{8}$ Another factor that may contribute to the macroscopic rheology of mixed polymer/surfactant systems is related to the dynamic nature of the surfactant micelles and of the surfactant clusters decorating the polymer backbone. The effect of the exchange of individual surfactant molecules between micelles and clusters, while difficult to assess, cannot be prevented, except by replacing the surfactant micelles by polymeric micelles.

Intrigued by the possibility of using polymeric micelles as surfactant substitutes in fluids containing cationic cellulose ethers, we undertook an investigation of polyelectrolyte complexes (PEC) consisting of a cationic derivative of hydroxyethylcellulose ether, JR400, and a copolymer of sodium 2-acrylamido2-methylpropanesulfonate and $N$ - $n$-dodecylmethacrylamide (PAMPS-Dod), which carries $\sim 5 \mathrm{~mol} \%$ of $n$ dodecyl moieties randomly distributed along the chain

${ }^{\dagger}$ To whom correspondence should be addressed (Phone: +1-514-343-6123, Fax: +1-514-343-2362, E-mail: francoise.winnik@umontreal.ca). 
Table I. Physical properties and structural features of the polymers

\begin{tabular}{lccccccc}
\hline Polymer & Charge & $\begin{array}{c}\text { Molecular } \\
\text { Weight }\end{array}$ & $\begin{array}{c}\text { Critical overlap } \\
\text { concentration } \\
\mathrm{c}^{*} / \mathrm{gL}^{-1}\end{array}$ & $\begin{array}{c}\text { Mean } \\
\text { contour } \\
\text { length/nm }\end{array}$ & $\begin{array}{c}\text { Mean contour } \\
\text { length between } \\
\text { charges } / \mathrm{nm}\end{array}$ & $\begin{array}{c}\text { Degree of } \\
\text { modification }\end{array}$ & $\begin{array}{c}\text { Charge } \\
\text { concentration in a } \\
10 \mathrm{~g} \mathrm{~L}^{-1} \text { solution }\end{array}$ \\
\hline JR400 & Positive & $500000^{\mathrm{a}}$ & $4.0^{\mathrm{e}}$ & $\sim 1000^{\mathrm{a}}$ & $10^{\mathrm{a}}$ & $27{\mathrm{~mol} \%\left(\mathrm{Me}_{3} \mathrm{~N}^{+}-\mathrm{R}^{\mathrm{a}}\right.}^{\mathrm{a}}$ & $10 \times 10^{-3} \mathrm{~mol} \mathrm{~L}^{-1}$ \\
PAMPS-Dod & Negative & $53000^{\mathrm{b}}$ & & 57 & 0.25 & $5 \mathrm{~mol} \%\left(n-\mathrm{C}_{12} \mathrm{H}_{25}\right)^{\mathrm{d}}$ & $41 \times 10^{-3} \mathrm{~mol} \mathrm{~L}^{-1}$ \\
PAMPS & Negative & $71000^{\mathrm{c}}$ & & 77 & 0.25 & - & $44 \times 10^{-3} \mathrm{~mol} \mathrm{~L}^{-1}$ \\
\hline
\end{tabular}

${ }^{\mathrm{a}}$ From ref 12. ${ }^{\mathrm{b}}$ From GPC (see experimental section). ${ }^{\mathrm{c}}$ From ref $14 .{ }^{\mathrm{d}}$ From H NMR spectrum, see ref 9. ${ }^{\mathrm{e}}$ From ref 15.

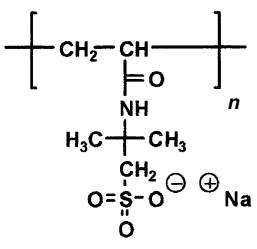

PAMPS

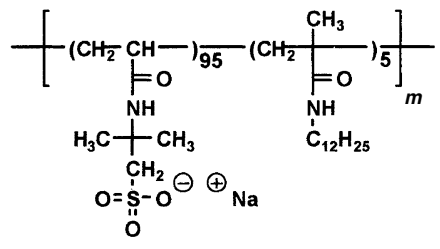

PAMPS-Dod

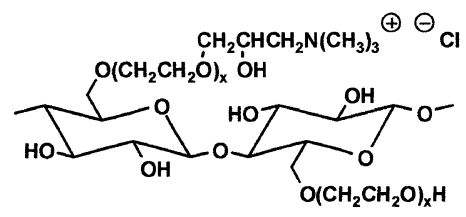

JR400

Figure 1. Structure of the polymers used in this study.

(Figure 1). The hydrophobically-modified polyanion, PAMPS-Dod, is known to associate in water, forming unimers $\sim 6 \mathrm{~nm}$ in diameter as well as larger aggregates approximately $120 \mathrm{~nm}$ in diameter that consist of a small number of assembled chains. ${ }^{9}$ Thus, conceptually, the JR400/PAMPS-Dod system presents similarities with a system consisting of JR400 and oppositely charged surfactant micelles. We reported previously the rheology of mixed systems consisting of small amounts of JR400 and excess PAMPS-Dod (polyanion-rich mixtures). The fluids in this concentration regime exhibit significant thixotropy as well as unexpected shear thickening behavior. ${ }^{10}$

We present here a rheological investigation of the same mixed system, but in the polyanion-poor concentration regime. We conducted oscillatory shear experiments to examine the effect of fluid composition on the complex viscosity, dynamic moduli and intersection frequency. Shear dependent viscosity measurements were conducted as well, to assess the validity of the Cox-Merz rule. The Carreau-Yasuda model was applied to characterize quantitatively the non-Newtonian properties exhibited by the mixed fluids investigated. Taken together, the results provide a strong basis in support of an interaction model that takes into account the differences in architecture, flexibility, and charge content of the two polyelectrolytes.

\section{EXPERIMENTAL}

\section{Materials}

Water was purified with a Millipore Milli-Q System. The polycation Ucare Polymer JR400 was obtained from Amerchol Corporation (Union Carbide) and was used as received. JR400 is the chloride salt of a trimethylammonium derivative of hydroxyethylcellulose (HEC) with a molecular weight of approximately $5 \times 10^{5}$ Dalton (Figure 1). ${ }^{11}$ The positive charge content of JR400 is $27 \mathrm{~mol} \%$, corresponding to 27 ammonium groups for every 100 glucose units (Table I). ${ }^{12}$ The polyanions PAMPS and PAMPS-Dod were prepared as described earlier. ${ }^{13,14}$ PAMPS-Dod is a copolymer of sodium 2-(acrylamido)-2-methylpropanesulfonate (AMPS) and $N$ - $n$-dodecylmethacrylamide (Dod) with a dodecyl content of $5 \mathrm{~mol} \%$. The molecular weight of PAMPS-Dod was determined to be approximately $5 \times 10^{4}$ Dalton by size exclusion chromatography (SEC) on a JASCO GPC-900 system equipped with Shodex Asahipak GF-7 M HQ columns using a $0.2 \mathrm{M}$ solution of $\mathrm{LiClO}_{4}$ in methanol as the eluent and was calibrated with poly(ethylene glycol) standards (Scientific Polymer Products, Inc.).

\section{Fluids for Rheology Measurements}

All solutions were prepared at ambient temperature. Stock solutions of each polymer, JR400, PAMPS or PAMPS-Dod $\left(10 \mathrm{~g} \mathrm{~L}^{-1}\right)$ were prepared by dissolution of the polymer in water for $12 \mathrm{~h}$ to allow complete hydration and swelling. The stock solutions were then gently stirred for $24 \mathrm{~h}$ and were allowed to equilibrate without stirring for another $24 \mathrm{~h}$ before use. Aqueous mixtures of JR400/PAMPS-Dod were prepared from weighed aliquots of stock solutions and mixed slowly. The mixtures were stirred for $48 \mathrm{~h}$. Special precautions were taken during the agitation to prevent the formation of air bubbles. The stirring speed was varied depending on the viscosity of the resulting mixture. Finally, the mixtures were allowed to stand at ambient temperature for $24 \mathrm{~h}$ prior to rheological measurement. The total polymer concentration of each mixture was $10 \mathrm{~g} \mathrm{~L}^{-1}$ (Table II). The ratio between the two compo- 
Table II. Characteristic of aqueous solutions of JR400/PAMPS-Dod mixtures

\begin{tabular}{ccccll}
\hline$f_{\mathrm{p}^{-}}$ & {$[\mathrm{JR} 400]$} & {$[$ PAMPS-Dod] } & [Total polymer] & Appearance & Property \\
\hline 0.00 & 10 & 0 & 10 & clear and homogeneous & viscous \\
0.01 & 9.9 & 0.1 & 10 & clear and homogeneous & viscous \\
0.02 & 9.8 & 0.2 & 10 & clear and homogeneous & viscous \\
0.03 & 9.7 & 0.3 & 10 & clear and homogeneous & gel-like \\
0.04 & 9.6 & 0.4 & 10 & clear and homogeneous & gel-like \\
0.05 & 9.5 & 0.5 & 10 & clear and homogeneous & gel-like \\
0.06 & 9.4 & 0.6 & 10 & clear and homogeneous & gel-like \\
0.08 & 9.2 & 0.8 & 10 & clear and homogeneous & gel-like \\
0.10 & 9.0 & 1.0 & 10 & turbid and homogeneous & gel-like \\
0.12 & 8.8 & 1.2 & 10 & turbid and homogeneous & gel-like \\
0.14 & 8.6 & 1.4 & 10 & precipitates in supernatant & viscous \\
\hline
\end{tabular}

nents in each mixture is expressed as the weight fraction of polyanion $\left(f_{\mathrm{p}^{-}}\right)$in the mixture (eq 1), where $W_{\mathrm{p}^{-}}$and $W_{\mathrm{p}^{+}}$are the weights of polyanion (PAMPS and PAMPS-Dod) and JR400 stock solutions, respectively.

$$
f_{\mathrm{p}^{-}}=\frac{W_{\mathrm{p}^{-}}}{W_{\mathrm{p}^{-}}+W_{\mathrm{p}^{+}}}
$$

The ratio between the two components in each mixture is expressed also as the charge concentration ratios $\left(c_{-} / c_{+}\right)$, where $c_{-}$is the molal concentration of negative charges contributed by the polyanion and $c_{+}$is the molal concentration of cationic charges contributed by JR400. Note that in a $10 \mathrm{~g} \mathrm{~L}^{-1}$ aqueous PAMPSDod solution, the negative charge concentration is 41 $\mathrm{mM}$ and the positive charge concentration of a $10 \mathrm{~g}$ $\mathrm{L}^{-1}$ aqueous JR400 solution is $10 \mathrm{mM}$. Charges from the counterions of polymers $\left(\mathrm{Cl}^{-}\right.$for $\mathrm{JR} 400$ or $\mathrm{Na}^{+}$for PAMPS-Dod) are not considered. The ionic strength of the samples was not adjusted, it varied depending on the ratio between the two components.

\section{Rheological Measurements}

Rheological measurements were performed at $25^{\circ} \mathrm{C}$ with a ReoLogica StressTech controlled stress rheometer, equipped with a NESLAB circulator to control the temperature within $\pm 0.1^{\circ} \mathrm{C}$. A concentric cylindrical measurement system (Bob and Cup) with a diameter of $25 \mathrm{~mm}$ was used for all measurements. Each sample was loaded into the cell with extreme care to prevent the formation of air bubbles and was allowed to rest for $15 \mathrm{~min}$ before measurement. The rheometer was set automatically to determine the shear viscosity $(\eta)$ with the applied shear rate $(\dot{\gamma})$ ranging between 0.01 and $785 \mathrm{~s}^{-1}$. Each scan started at low shear rate $\left(0.01 \mathrm{~s}^{-1}\right)$ with the shear rate increasing steadily toward higher values. Immediately after reaching the highest attainable shear rate for each sample, the measurement was continued with decreasing shear rates. The time interval between $\eta$ measurements at each shear rate $(\Delta t)$ was set at $10 \mathrm{~s}$ for all samples. Most of the oscillatory mea- surements were conducted within the linear viscoelastic region, where the dynamic storage modulus $\left(G^{\prime}\right)$ and loss modulus $\left(G^{\prime \prime}\right)$ are independent of the applied stress. Oscillation stress sweeps were performed at various frequencies to determine the stress amplitude. A delay time of $10 \mathrm{~s}$ and an integration time of $1 \mathrm{~s}$ were employed for all oscillatory measurements. Oscillatory measurements in the frequency range $0.001-10 \mathrm{~Hz}$ are reported.

\section{RESULTS AND DISCUSSION}

\section{Physical Appearance of Mixed Solutions}

Samples for analysis were prepared with various weight fractions of PAMPS-Dod $\left(f_{\mathrm{p}^{-}}\right)$by mixing stock solutions $\left(10 \mathrm{~g} \mathrm{~L}^{-1}\right)$ of JR400 and PAMPS-Dod. Precipitation occurred immediately upon combining the stock solutions, a common occurrence when mixing oppositely charged polyelectrolytes. However upon prolonged stirring, the precipitate gradually disappeared in several samples that became clear, homogeneous fluids. The composition and appearance of the fluids studied are listed in Table II. Another consequence of mixing the two polymer solutions was a significant increase in viscosity, as depicted in Figure 2, which presents the composition-dependant viscosity of mixed fluids composed of JR400 and either PAMPS-Dod or PAMPS. The viscosity of mixed JR400/PAMPS-Dod samples was enhanced up to 2000 times, compared to that of a $10 \mathrm{~g} \mathrm{~L}^{-1}$ solution of JR400, but mixed JR400/PAMPS solutions experienced only a weak increase in viscosity, compared to the starting solutions. The viscosity effects strongly suggest that the interactions between JR400 and PAMPS-Dod in water are of a different nature than typical polyanion/polycation interactions. This observation prompted us to conduct further rheological measurements in order to unravel the interaction mechanism. 


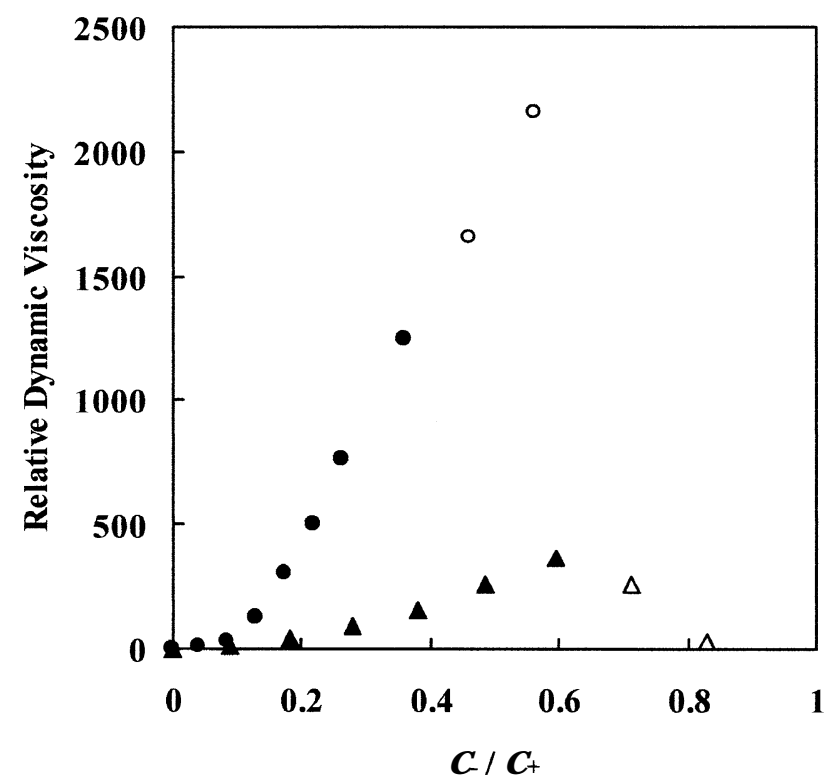

Figure 2. Plots of the relative dynamic viscosity measured at $0.01 \mathrm{~Hz}$ as a function of $c_{-} / c_{+}$, the charge ratio between PAMPS (triangle) or PAMPS-Dod (circle) and JR400 in $10 \mathrm{~g} \mathrm{~L}^{-1}$ aqueous mixtures of JR400/PAMPS and JR400/PAMPS-Dod. The full symbols represent clear homogeneous mixtures and the open symbols indicate turbid homogeneous mixtures.

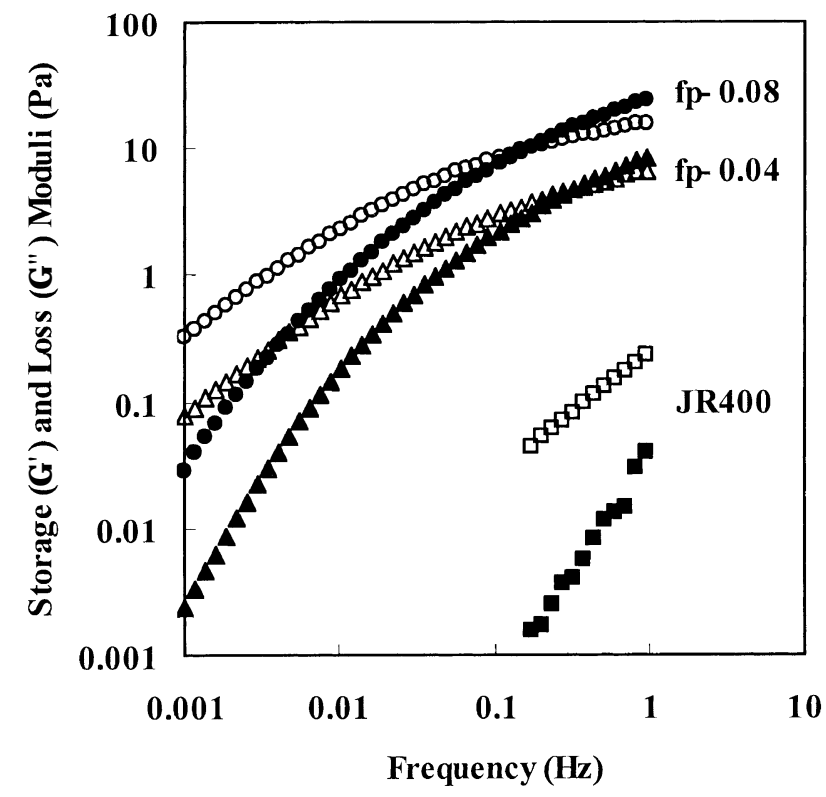

Figure 3. Frequency dependence of the storage modulus $G^{\prime}$ (full symbols) and the loss modulus $G^{\prime \prime}$ (open symbols) for a $10 \mathrm{~g}$ $\mathrm{L}^{-1}$ aqueous solution of JR400 (squares) and for $10 \mathrm{~g} \mathrm{~L}^{-1}$ aqueous solutions of JR400/PAMPS-Dod mixtures with $f_{\mathrm{p}^{-}} 0.04$ (triangles) and 0.08 (circles).

\section{Storage Modulus $\left(G^{\prime}\right)$ and Loss Modulus $\left(G^{\prime \prime}\right)$}

Oscillatory shear experiments were carried out next on all homogeneous mixed fluids to gather data on the frequency dependence of the storage modulus $\left(G^{\prime}\right)$ and the loss modulus $\left(G^{\prime \prime}\right)$ of the fluids. Profiles of $G^{\prime}$ and $G^{\prime \prime}$ are presented in Figure 3, for fluids of $f_{\mathrm{p}^{-}} 0.04$ and 0.08 , as well as for a solution of JR400. All mixed

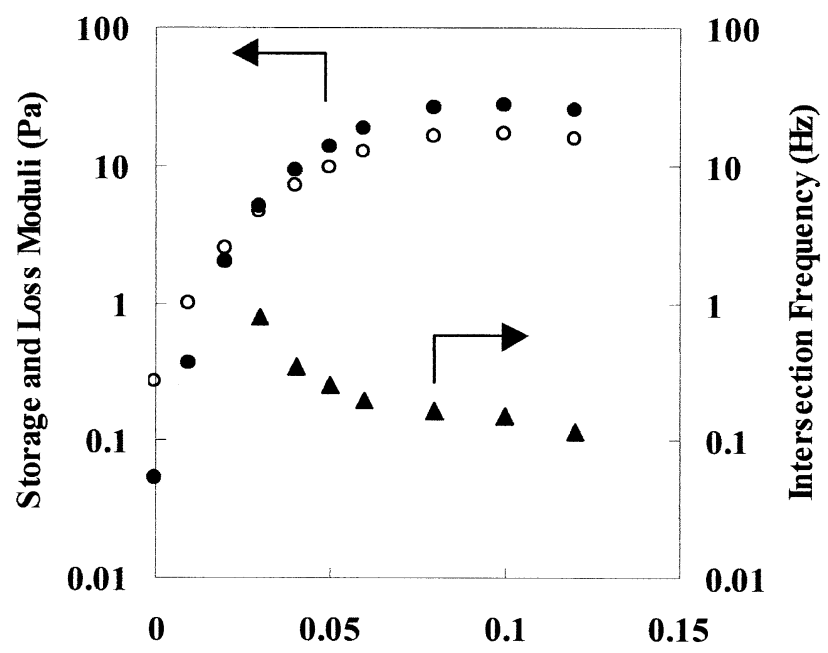

Weight Fraction of Polyanion in Mixtures, $\mathrm{fp}$ -

Figure 4. Storage modulus $G^{\prime}$ (full circles), loss modulus $G^{\prime \prime}$ (open circles) and intersection frequency (triangles), the frequency at which $G^{\prime}$ equals $G^{\prime \prime}$, plotted as a function of $f_{\mathrm{p}^{-}}$, the weight fraction of PAMPS-Dod in $10 \mathrm{~g} \mathrm{~L}^{-1}$ aqueous mixtures of JR400/PAMPS-Dod. The dynamic moduli were measured at a frequency of $1 \mathrm{~Hz}$.

fluids of $f_{\mathrm{p}^{-}}>0.02$ exhibited viscoelastic behavior: in the low frequency domain, the mixtures are viscous $\left(G^{\prime \prime}>G^{\prime}\right)$ while at frequencies higher than a certain threshold value the elastic response dominates $\left(G^{\prime}>\right.$ $\left.G^{\prime \prime}\right)$. We confirmed that aqueous solutions of JR400 in the absence of PAMPS-Dod are typical Newtonian fluids with $G^{\prime} \sim \omega^{2}, G^{\prime \prime} \sim \omega^{1}$, and $G^{\prime \prime}>G^{\prime}$ over the entire frequency domain studied $(0.001-10 \mathrm{~Hz})$ (Figure 3). The weak elastic response of the JR400 solution reflects the formation of a transient network created via interchain entanglements, since the concentration of the solution is well above the dilute regime $\left(<4.0 \mathrm{~g} \mathrm{~L}^{-1}\right) .{ }^{15}$ Attempts were made to fit the data plotted in Figure 3 for the mixed fluids to the single-mode Maxwell model which is commonly applied to characterize viscoelastic fluids and to describe the frequency dependence of $G^{\prime}$ and $G^{\prime \prime}$. No satisfactory fit could be obtained, which should not surprise, as it is a known fact that the Maxwell data analysis is rarely adequate to model associating polymers or two-component systems. ${ }^{16,17}$ The ineptness of the single-mode Maxwell model hints at the complexity of the JR400/PAMPSDod system.

It can be seen also in Figure 3 that the crossover frequency for the fluid of $f_{\mathrm{p}^{-}} 0.08$ is lower than that observed for the fluid containing less PAMPS-Dod $\left(f_{\mathrm{p}^{-}}\right.$ $0.04)$. This is a general trend, the lower the polyanion content, the higher the crossover frequency, as seen in Figure 4, where the crossover frequency of mixed samples is plotted as a function of fluid composition. Thus the relaxation time $\tau$ of the fluid $(\tau=1 / \omega)$ is shifted 


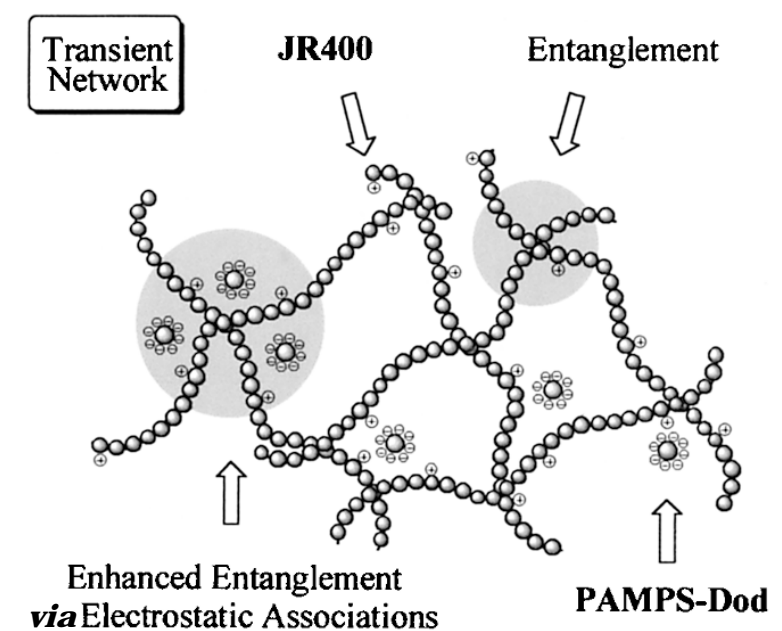

Figure 5. Schematic representation of the transient network for the interpolyelectrolyte complexes formed in the clear homogeneous fluids.

towards longer times as the PAMPS-Dod concentration in the sample increases. A similar observation has been reported in a study of mixed JR400/surfactants systems. ${ }^{7}$ The slowing down of the system dynamics with increasing polyanion concentration is indicative of the formation of a network of increasing crosslinking density and junction lifetimes. Figure 4 also presents the changes with fluid composition of $G^{\prime}$ and $G^{\prime \prime}$ at a given frequency $(1 \mathrm{~Hz})$. Both moduli increase sharply with increasing PAMPS-Dod concentration, reaching values $\sim 500$ times higher than the values registered for a solution of JR400. Maximum elastic modulus enhancement is reached for fluids of $f_{\mathrm{p}^{-}} \sim 0.1$. Increasing the polyanion concentration beyond this value has practically no effect on either the moduli or the crossover frequency.

The $G^{\prime}, G^{\prime \prime}$, and the crossover frequency, which all three reflect the cross-linking density of a viscoelastic system, concur in favor of the interaction model presented in Figure 5, based on the structural differences between the two oppositely charged polyelectrolytes. Although JR400 is only lightly charged $(27 \mathrm{~mol} \%$ positive charges), it exists in a stretched conformation as a result of the rigidity of its polysaccharide backbone. In contrast, PAMPS-Dod forms compact unimolecular micelles, $\sim 6 \mathrm{~nm}$ in diameter. Thus it is possible for a single highly charged PAMPS-Dod unimer to associate with two or more JR400 chains and consequently to act as crosslinking point of a network composed predominantly of entangled rigid polycation chains. Increasing the weight fraction of PAMPS-Dod results in an increase in crosslinking density, until a saturation concentration is reached where further polyanion addition does not generate new joining points and macroscopic phase separation occurs. This interaction model, suggested by the oscillatory shear experiments and the physical ap- pearance of the fluids, was confirmed by measurements of the shear flow viscosity described next.

\section{Shear Flow Viscosity}

Figure 6 displays the steady shear viscosity $(\eta)$ as a function of shear rate for mixed fluids of $f_{\mathrm{p}^{-}} 0.01$, 0.02 , and 0.04 . It can be seen that the three mixtures exhibit a Newtonian regime at low shear rates but they become shear thinning in the high shear rate domain. With increasing polyanion content, the viscosity of the mixtures increases and the onset of the shear thinning regime shifts to lower shear rates. Figure 6 also gives the corresponding variations of the dynamic complex viscosity $(\eta *)$ with frequency. In the Newtonian regime, the $\eta *$ and $\eta$ profiles coincide and, as expected, $\eta *$ approaches the steady-flow viscosity $\left(\eta_{0}\right)$ at low frequencies. However, at high frequencies or shear rates, the values of $\eta$ and $\eta *$ diverge, with $\eta$ consistently higher than $\eta *$. The difference between $\eta$ and $\eta *$ increases with shear rate and it becomes more pronounced as the PAMPS-Dod content of the fluids increases, as seen if one compares Figure 6 , top, $\left(f_{\mathrm{p}^{-}}\right.$ $0.01)$ and Figure 6, bottom $\left(f_{\mathrm{p}^{-}} 0.04\right)$.

In solutions of a polymer in the entangled regime or in polymer melts, it is usually observed that, for identical values of the oscillatory frequency and the shear rate, $\eta *$ equals $\eta$ over the entire frequency range, an observation commonly known as the Cox-Merz rule. ${ }^{18,19}$ Departure from the rule is taken as an indication that long range interactions occur and provides supportive, indirect, evidence for the presence of aggregates. Therefore, the invalidity of the Cox-Merz rule observed here (Figure 6) confirms the formation of a network-like structure in the JR400/PAMPS-Dod fluids. A similar observation was reported by Chronakis and Alexandridis in their rheological study of the interactions between JR400 and surfactants. ${ }^{7}$

\section{Carreau-Yasuda Model}

The non-Newtonian properties of the aqueous JR400/PAMPS-Dod mixtures were further examined by fitting each shear viscosity profile, as a function of shear rate, with the Carreau-Yasuda equation (eq 2): ${ }^{20}$

$$
\eta=\eta_{0}\left[1+(\lambda \dot{\gamma})^{a}\right]^{-d / a}
$$

where $\eta$ represents the shear viscosity, $\eta_{0}$ the zero-shear viscosity, $\dot{\gamma}$ the shear rate, and $\lambda$ a time constant which is equal to the inverse of the critical shear rate corresponding to the onset of shear thinning and is related to the relaxation time of the system. The dimensionless parameter $a$ describes the transition region between the Newtonian region and the power-law region while $d$, the negative of the power-law slope, shows directly the 

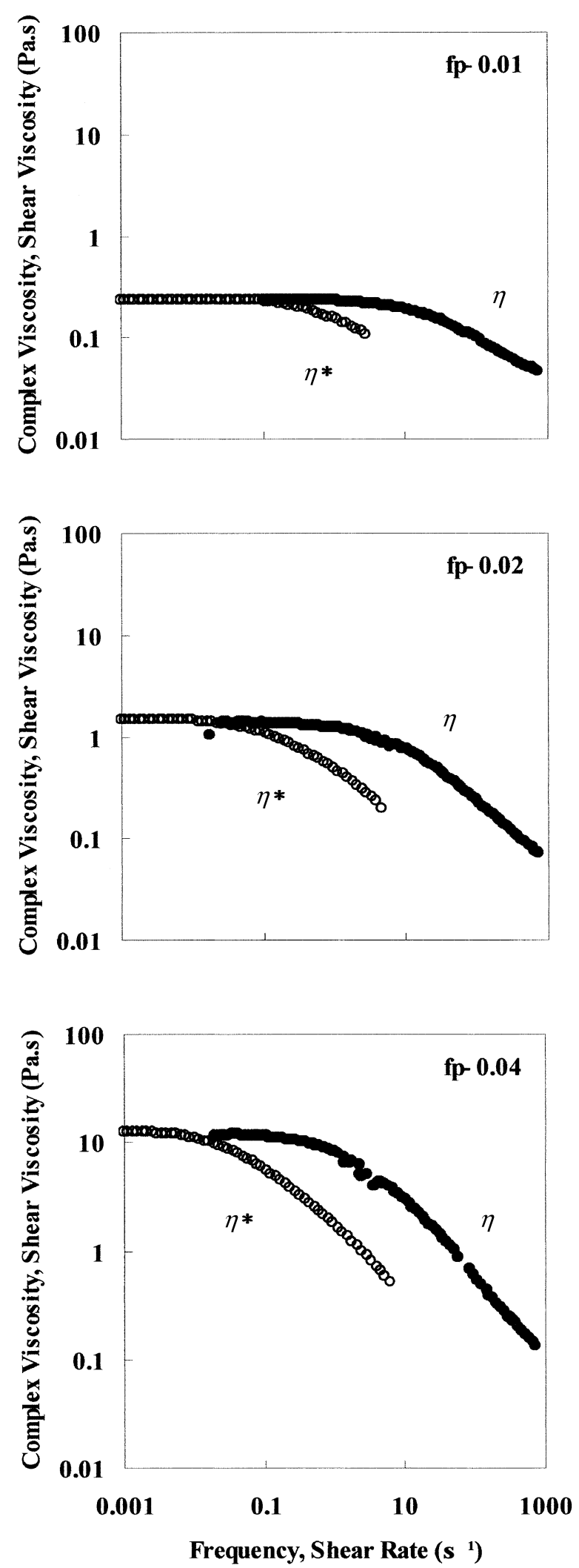

Figure 6. Steady shear viscosity $(\eta)$ and dynamic complex viscosity $(\eta *)$ as a function of shear rate or frequency for $10 \mathrm{~g} \mathrm{~L}^{-1}$ aqueous solutions of JR400/PAMPS-Dod mixtures of various compositions.

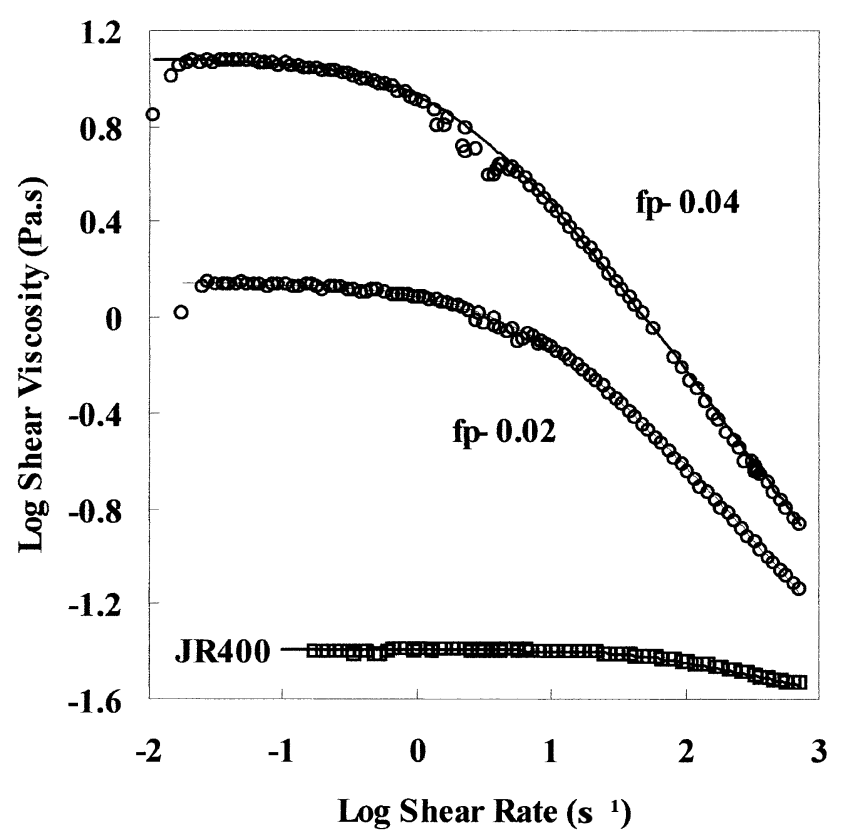

Figure 7. Steady shear viscosity as a function of shear rate for $10 \mathrm{~g} \mathrm{~L}^{-1}$ aqueous solutions of JR400/PAMPS-Dod mixtures with $f_{\mathrm{p}^{-}} 0.02$ and 0.04 and for a $10 \mathrm{~g} \mathrm{~L}^{-1}$ aqueous solution of JR400. The solid lines represent the best fits with the Carreau-Yasuda equation.

Table III. Best-fit parameters obtained from Carreau-Yasuda equation for the rheological data recorded with aqueous solutions $\left(10 \mathrm{~g} \mathrm{~L}^{-1}\right)$ of JR400/PAMPS-Dod mixtures of various

\begin{tabular}{crccc}
\multicolumn{5}{c}{ compositions } \\
\hline Sample $f_{\mathrm{p}^{-}}$ & $\eta_{0} / \mathrm{Pa} . \mathrm{s}$ & $\lambda / \mathrm{s}$ & $a$ & $d$ \\
\hline 0.00 & 0.04 & 0.02 & 1.38 & 0.22 \\
0.01 & 0.24 & 0.04 & 0.83 & 0.51 \\
0.02 & 1.39 & 0.13 & 0.83 & 0.66 \\
0.03 & 4.10 & 0.25 & 0.95 & 0.71 \\
0.04 & 12.10 & 0.50 & 0.90 & 0.76 \\
0.05 & 19.60 & 0.55 & 0.80 & 0.78 \\
0.06 & 26.60 & 0.58 & 0.88 & 0.80 \\
0.08 & 44.00 & 0.70 & 0.80 & 0.84 \\
0.10 & 78.00 & 0.80 & 0.50 & 0.86 \\
0.12 & 100.00 & 1.00 & 0.40 & 0.90 \\
\hline
\end{tabular}

steepness of the power-law region.

The Carreau-Yasuda model gave excellent fits to the shear induced changes in viscosity of the mixed JR400/PAMPS-Dod fluids, as shown in Figure 7 for the mixed fluids of $f_{\mathrm{p}^{-}} 0.02$ and 0.04 and for a JR400 solution. The parameters derived from the fits for all mixed fluids are given in Table III. The increase in the parameter $\lambda$ with increasing $f_{\mathrm{p}^{-}}$can be taken as an indication of the increase in the size and number of crosslinking points between JR400 chains as more PAMPS-Dod micelles are added to the mixtures. Turning to the parameter $d$, which also increases with increasing $f_{\mathrm{p}^{-}}$, it is useful to recall that, in his studies of linear and star polystyrene of different molecular weights, Yasuda 
demonstrated that $d$ depends on the molecular weight of the polymer, but not on its shape. ${ }^{20}$ Thus, the increase in the parameter $d$ with increasing PAMP-Dod provides yet additional support to the JR400/PAMPS-Dod model proposed here, since it can be assigned to an increase in the apparent molecular weight of the JR400/PAMPSDod complex as more and more anionic crosslinking agents are added, even though the total polymer concentration remains constant and the molecular weight of the individual components remain unchanged.

\section{CONCLUSION}

We have reported here the preparation of clear homogeneous viscoelastic aqueous fluids composed of a stretched rigid lightly charged polycation and a compact flexible highly charged polyanion. Steady shear and oscillatory shear measurements show that the nonNewtonian properties of the aqueous mixtures are dictated primarily by the relative amounts of the two oppositely charged polyelectrolytes at constant total polymer concentration. The rheological study supports an association model involving interpolyelectrolyte complexes with a network-like structure, based on electrostatic interactions between two oppositely charged polyelectrolytes of grossly unmatched size, rigidity, and charge density. The Cox-Merz rule did not apply to the rheological data, however the non-Newtonian behavior of the JR400/PAMPS-Dod mixtures could be fitted with the empirical Carreau-Yasuda equation, providing the time constants associated to the relaxation process. The increase, with increasing PAMPS-Dod content, of the parameter $d$ of the Carreau-Yasuda model, the zero shear viscosity, the dynamic moduli, and the relaxation time, suggests that increasing the weight fraction of PAMPS-Dod in mixtures enhances the extent of the interpolymeric network, accounting for the amplification of the viscosity and viscoelasticity effects.

Acknowledgment. This work was supported in part by the Shorai Foundation for Science and Technology and by a grant from the Natural Science and Engineering Research Council of Canada to F. M. Winnik. R. C. W. Liu thanks the Association of International Education, Japan, (AIEJ) for a Short-term Exchange Promo tion Program (Inbound) Scholarship to support his stay in Osaka University.

\section{REFERENCES}

1. E. D. Goddard, in "Principles of Polymer Science and Technology in Cosmetics and Personal Care", E. D. Goddard and J. V. Gruber, Ed., Marcel Dekker, Inc., New York, N.Y., 1999, p 113.

2. J. E. Glass, Ed., "Polymers in Aqueous Media", Advances in Chemistry Series No. 213, American Chemical Society, Washington, D.C., 1989.

3. D. N. Schultz and J. E. Glass, "Polymers as Rheology Modifiers", ACS Symposium Series, No. 462, American Chemical Society, Washington. D.C., 1991.

4. B. Jonsson, B. Lindman, K. Holmberg, and B. Kronberg, Surfactants and Polymers in Aqueous Solutions", John. Wiley \& Sons Ltd., Chichester, 1998.

5. P. S. Leung, E. D. Goddard, C. Han, and C. J. Glinka, Colloids Surf., 13, 47 (1985).

6. U. Kästner, H. Hoffmann, R. Dönges, and R. Ehrler, Colloids Surf. A, 112, 209 (1996).

7. I. S. Chronakis and P. Alexandridis, Macromolecules, 34, 5005 (2001).

8. M. M. Guerrini, I. I. Negulescu, and W. H. Daly, J. Appl. Polym. Sci., 68, 1091 (1998).

9. A. Hashidzume, H. Yamamoto, M. Mizusaki, and Y. Morishima, Polym. J., 31, 1009 (1999).

10. R. C. W. Liu, Y. Morishima, and F. M. Winnik, Macromolecules, 34, 9117 (2001).

11. S. Dhoot, E. D. Goddard, D. S. Murphy, and M. Tirrell, Colloids Surf., 66, 91 (1992).

12. O. Regev, E. F. Marques, and A. Khan, Langmuir, 15, 642 (1999).

13. Y. Morishima, Y. Tominaga, M. Kamachi, T. Okada, Y. Hirata, and N. Mataga, J. Phys. Chem., 95, 6027 (1991).

14. M. Mizusaki, Y. Morishima, and F. M. Winnik, Macromolecules, 32, 4137 (1999).

15. M. Tsianou, Ph.D. Thesis in Physical Chemistry, Lund University, Lund, Sweden, 1999.

16. K. C. Tam, R. D. Jenkins, M. A. Winnik, and D. R. Bassett, Macromolecules, 31, 4149 (1988).

17. S. Deguchi, K. Kuroda, K. Akiyoshi, B. Lindman, and J. Sunamoto, Colloids Surf. A, 147, 203 (1999).

18. W. W. Graessley, Adv. Polym. Sci., 16, 1 (1974).

19. R. G. Larson, "The Structure and Rheology of Complex Fluids”, Oxford University Press, Inc., London, 1999.

20. K. Yasuda, R. C. Armstrong, and R. E. Cohen, Rheol. Acta, 20, 163 (1981). 